\title{
Non-invasive prenatal testing for detection of Down syndrome in Government-funded projects: a cost-effectiveness analysis based on real-world data from China
}

\author{
Wenru Shang ${ }^{1}$, Yang Wan ${ }^{2}$, Jianan $\mathrm{Chen}^{3}$, Yanqiu Du ${ }^{1}$, and jiayan Huang ${ }^{1}$ \\ ${ }^{1}$ Fudan University \\ ${ }^{2}$ Fuyang People's Hospital \\ ${ }^{3}$ University of Oslo
}

May 5, 2020

\begin{abstract}
Abstract: Objective: This study aimed to assess the cost-effectiveness of implementing NIPT screening for DS based on government-funded projects in China. Design: Decision analytical model. Setting: Fuyang City People's Hospital (FCPH) in China. Population: 1,400 maternal women in FCPH for the period March to June 2019. Methods: An incremental costeffectiveness ratio (ICER) was calculated from a societal perspective. Univariate sensitivity analysis measured the impact of the uptake rate of NIPT and the cost of NIPT, and probabilistic sensitivity analysis (PSA) also was conducted. Main outcome measures:The number of DS case detected. Results: NIPT age-related screening strategy (Strategy D) has the lowest ICER, with an ICER of USD 25,641.22 per additional DS case detected compared with second-trimester serum screening Strategy (Strategy A). Universal NIPT screening (Strategy E) was the most effective and showed more DS case detected compared with alternative strategies. However, it was also the most expensive strategy. Strategy E would not be cost-effective unless the unit cost of NIPT could be decreased to USD 57.00. PSA results indicated that, at willingness-to-pay threshold under USD 120,000.00, Strategy D is cost-effective compared with combined serum and NIPT screening (Strategy C, also the referred strategy in China). Conclusion: Compared with current referred screening strategy in China, age-related NIPT contingent screening performances better in cost-effectiveness analysis and safety, which should be recommended to pregnant women. While under the government-funded projects in China, universal NIPT screening is still not optimal. Lowering the price of NIPT is an effective measure to promote universal NIPT Strategy
\end{abstract}

\section{Hosted file}

Main Document.doc available at https://authorea.com/users/302205/articles/432228-noninvasive-prenatal-testing-for-detection-of-down-syndrome-in-government-funded-projectsa-cost-effectiveness-analysis-based-on-real-world-data-from-china

\section{Hosted file}

Figure file.docx available at https://authorea.com/users/302205/articles/432228-non-invasiveprenatal-testing-for-detection-of-down-syndrome-in-government-funded-projects-a-costeffectiveness-analysis-based-on-real-world-data-from-china 